\title{
Mechanical signals control SOX-9, VEGF, and c-Myc expression and cell proliferation during inflammation via integrin-linked kinase, B-Raf, and ERK1/2-dependent signaling in articular chondrocytes
}

\author{
Priyangi M Perera1, Ewa Wypasek1, Shashi Madhavan', Birgit Rath-Deschner², Jie Liu', Jin Nam¹, Bjoern Rath³, \\ Yan Huang ${ }^{1}$, James Deschner ${ }^{4}$, Nicholas Piesco5 ${ }^{5}$, Chuanyue Wu6 and Sudha Agarwal*
}

\begin{abstract}
Introduction: The importance of mechanical signals in normal and inflamed cartilage is well established. Chondrocytes respond to changes in the levels of proinflammatory cytokines and mechanical signals during inflammation. Cytokines like interleukin (IL)-1 $\beta$ suppress homeostatic mechanisms and inhibit cartilage repair and cell proliferation. However, matrix synthesis and chondrocyte (AC) proliferation are upregulated by the physiological levels of mechanical forces. In this study, we investigated intracellular mechanisms underlying reparative actions of mechanical signals during inflammation.

Methods: ACs isolated from articular cartilage were exposed to low/physiologic levels of dynamic strain in the presence of IL-1 $\beta$. The cell extracts were probed for differential activation/inhibition of the extracellular signalregulated kinase 1/2 (ERK1/2) signaling cascade. The regulation of gene transcription was examined by real-time polymerase chain reaction.
\end{abstract}

Results: Mechanoactivation, but not IL-1 $\beta$ treatment, of ACs initiated integrin-linked kinase activation. Mechanical signals induced activation and subsequent C-Raf-mediated activation of MAP kinases (MEK1/2). However, IL-1 $\beta$ activated B-Raf kinase activity. Dynamic strain did not induce B-Raf activation but instead inhibited IL-1 $\beta$-induced B-Raf activation. Both mechanical signals and IL-1 $\beta$ induced ERK1/2 phosphorylation but discrete gene expression. ERK1/2 activation by mechanical forces induced SRY-related protein-9 (SOX-9), vascular endothelial cell growth factor (VEGF), and c-Myc mRNA expression and AC proliferation. However, IL-1 $\beta$ did not induce SOX-9, VEGF, and c-Myc gene expression and inhibited AC cell proliferation. More importantly, SOX-9, VEGF, and Myc gene transcription and AC proliferation induced by mechanical signals were sustained in the presence of IL-1 $\beta$.

Conclusions: The findings suggest that mechanical signals may sustain their effects in proinflammatory environments by regulating key molecules in the MAP kinase signaling cascade. Furthermore, the findings point to the potential of mechanosignaling in cartilage repair during inflammation.

\section{Introduction}

Mechanical loading during joint movement is critical for cartilage function and survival. Chondrocytes located

* Correspondence: agarwal.61@osu.edu

1 Biomechanics and Tissue Engineering Laboratory, The Ohio State University, Postle Hall, 305 W 12th Avenue, Columbus, OH 43210, USA

Full list of author information is available at the end of the article within the cartilage recurrently experience mechanical forces during joint movements. These cells sense, interpret, and respond to mechanical signals to maintain tissue integrity and homeostasis [1-5]. Activation of cells by mechanical signals is a rapid process and leads to activation of several intracellular signaling cascades, flow channels, and genes [6-8]. Accumulating evidence suggests 
that chondrocytic mechanosensing is discriminatory and capable of recognizing and responding to signals of various magnitudes to differentially regulate cartilage repair and pathologies $[4,9]$.

Similarly to soluble ligands, mechanotransduction is initiated at the matrix-membrane interface $[10,11]$. Chondrocytes located in the extracellular matrix are believed to relay mechanical signals through the plasma membrane via integrins [12,13]. Integrin-linked kinase (ILK), located in the cytoplasmic domain of integrins, plays a key role in transmitting mechanical signals to the intracellular compartment [13-15]. Within the cells, Ras (p21), Rho, and Rac belonging to the GTPase family of proteins are stimulated following activation of ILK and certain growth factor receptors [16,17]. Ras activation via exchange of guanosine diphosphate (GDP) to guanosine triphosphate (GTP) allows Ras to bind proto-oncogene cRAF kinases (Rafs) via Ser/Thr/Tyr phosphorylation of A-Raf, B-Raf, and c-Raf at multiple sites [18]. Phosphorylated Rafs activate mitogen-activated protein kinase (MAPK) kinase (MEK1/2) by phosphorylation of Ser217/ Ser221 [19]. Subsequently, MEK1/2 activates extracellular receptor kinase $1 / 2 \quad$ (ERK1/2) by phosphorylating Thr202/Tyr204. ERK1/2 activation is associated with growth signals. However, cytokines like interleukin-1 (IL1 ) and tumor necrosis factor-alpha (TNF- $\alpha$ ) also phosphorylate ERK1/2 to regulate certain proinflammatory genes $[20,21]$. Following activation, ERK1/2 translocates to the nucleus and activates transcription factors that are specific to the signals perceived by cells [22].

During inflammation, chondrocytes are exposed to proinflammatory cytokines such as IL- $1 \beta$ and TNF- $\alpha$. These cytokines alter their chondrogenic potential, prevent cell proliferation, and induce dedifferentiation and apoptosis. Specifically, cells exposed to IL-1 $\beta$ lose their ability to express SRY-related protein-9 (SOX-9) and vascular endothelial cell growth factor (VEGF) [23]. However, mechanical signals are shown to be reparative and upregulate proliferation and expression of collagen type II and proteoglycans in articular chondrocytes (ACs). These signals activate ERK1/2, suggesting a role for this signaling cascade in cartilage repair [12,24]. In this study, we investigated the intracellular signaling events responsible for beneficial/reparative effects of mechanical signals during inflammation. We demonstrate that mechanical signals and IL- $1 \beta$ both regulate the ERK1/2 signaling cascade but lead to activation of disparate transcription factors and gene expression. Strikingly, the actions of mechanical signals are sustained in the inflammatory environment and upregulate SOX-9, VEGF, and c-Myc gene transcription as well as chondrocyte proliferation.

\section{Materials and methods \\ Cell isolation, culture, and exposure to dynamic tensile or compressive forces}

ACs were isolated from knee joints of 12- to 14-week-old, female, Sprague Dawley rats (Charles River Laboratories, Inc., Wilmington, MA, USA) as described earlier. Briefly, cartilage from the condyles of femurs and tibia were aseptically removed, chipped, and digested in $1,400 \mathrm{U} / \mathrm{mL}$ collagenase type I (Worthington Biochemical Corporation, Lakewood, NJ, USA) for 3 hours at $37^{\circ} \mathrm{C}$. The cells were washed and grown in medium (tissue culture medium, or TCM) containing Ham's F12, 10\% fetal bovine serum (FBS), $10 \mathrm{U}$ penicillin, $10 \mu \mathrm{g} / \mathrm{mL}$ streptomycin, and $2 \mathrm{mM}$ glutamine (Invitrogen Corporation, Carlsbad, CA, USA). Cells were used in the first three passages.

ACs were subjected to dynamic tensile forces (dynamic strain, or DS) as described previously [3,25]. Briefly, ACs $\left(6 \times 10^{4} / 3 \mathrm{~mL}\right.$ TCM per well $)$ were plated in Bioflex plates (Flexcell International Corporation, Hillsborough, NC, USA) and cultured for 5 days to attain $70 \%$ to $80 \%$ confluence. Subsequently, 18 hours prior to exposing cells to DS or IL-1 $\beta$, the medium was replaced with TCM containing $1 \%$ FBS. Cells were exposed to DS at a magnitude of $6 \%$ and $0.25 \mathrm{~Hz}$ for the required time interval and the mRNA or proteins were extracted as described below.

\section{Western blot analysis}

Western blot assays were performed as described previously. Briefly, AC cells were lysed in Ripa buffer (Santa Cruz Biotechnology, Inc., Santa Cruz, CA, USA) containing protease and phosphatase inhibitor cocktail-2 (Sigma-Aldrich, St. Louis, MO, USA). The cell lysates were subjected to SDS-10\%-PAGE, electrotransferred to a nitrocellulose membrane (Bio-Rad Laboratories, Inc., Hercules, CA, USA), and reacted with antibodies to phospho-Thr202/Tyr204 ERK1/2 and total ERK1/2, phospho-Ser 217/221 MEK1/2 and total MEK1/2, phospho-Ser338 cRaf, phospho-Ser445 B-Raf, phosphoThr423-PAK1, phospho-Thr58/Ser62 Myc, and total cMyc proteins (Cell Signaling Technology, Inc., Danvers, MA, USA). Protein loading was normalized with total $\beta$ actin or antibodies to total signaling molecule in each sample. The primary antibodies were probed with horseradish peroxidase (HRP)- or IR-Dye 680- or IR-Dye-880conjugated secondary antibodies and scanned using a Kodak 1000 Image Documentation System (Eastman Kodak Company, Rochester, NY, USA) for HRP or an Odyssey infrared imaging system (LI-COR Biosciences, Lincoln, NE, USA) for IR-Dye-labeled antibodies. In some experiments, cells were pretreated with various inhibitors such as ERK inhibitor PD98059 (SABiosciences Corporation, Frederick, MD, USA) or Ras inhibitor GGT12133 (Pierce, Rockford, IL, USA) at the specified 
concentrations 30 minutes prior to mechanoactivation or IL-1 treatment or both.

\section{RAS activation}

The activated RAS in cells was estimated with an Active Ras Pull-Down and Detection Kit (Pierce) in accordance with the manufacturer's recommended protocol. Briefly, glutathione-S-transferase (GST) fusion protein containing the Ras-binding domain (RBD) of Raf1 (GST-Raf1$\mathrm{RBD}$; approximately $42 \mathrm{kDa}$ ) was incubated with cell lysate and glutathione agarose beads. The active Ras bound to the GST-Raf1-RBD was pulled down by centrifugation, and active RAS was detected by Western blot analysis using anti-Ras antibody. Control reactions using GTP $\gamma$ and GDP were performed to ensure that only active RAS was bound to GTP.

\section{Real-time polymerase chain reaction}

Total RNA was extracted with an RNeasy Micro Kit (Qiagen Inc., Valencia, CA, USA), and real-time polymerase chain reaction (RT-PCR) was conducted as described earlier [3]. Gene-specific primers used to amplify the cDNA (SYBR Green Master Mix; Bio-Rad Laboratories, Inc.) were rat VEGF (sense) GCCTTGTTCAGAGCGGAGAAA and (anti-sense) CGCGAGTCTGTGTTTTTGCA, rat MYC (sense) GGAAAACAACGAAAAGGCCC and (antisense) TGCTCATCTGCTTGAACGGAC, and rat SOX-9 (sense) ATCTGAAGAAGGAGAGCGAG and (antisense) CAAGCTCTGGAGACTGCTGA. Collected data were analyzed by the comparative threshold cycle method [26].

\section{Cell proliferation assay}

The cell proliferation was examined over a 3-day period by the MTT (3-(4,5 dimethylthiazolyl-2)-2,5-diphenyltetrazolium bromide) cell proliferation assay (American Type Culture Collection, Manassas, VA, USA) in accordance with the manufacturer's recommended protocol. The cells following treatment were incubated for 3 hours with $100 \mu \mathrm{L} / \mathrm{mL}$ MTT $(5 \mathrm{mg} / \mathrm{mL}$ Hanks' balanced salt solution), and the formazan formation was assessed by absorbance at $450 \mathrm{~nm}$ (Victor Plate Reader; PerkinElmer Inc., Waltham, MA, USA). The cell proliferation was calculated as mean absorbance of cells exposed to DS divided by mean absorbance of controls.

\section{Transfection of ACs with wild-type and mutant forms of FLAG-tagged ILK}

To examine the role of ILK in ERK1/2 activation, ACs were transfected with FLAG (polypeptide protein sequence DYKDDDDK)-ILK expression vectors, which were kindly provided by Chuanyue $\mathrm{Wu}$, of the University of Pittsburgh (Pittsburgh, PA, USA). ACs grown to $70 \%$ confluence were transfected with various expression plasmids containing wild-type (WT) ILK cDNA (residues 1 to 145; pFLAG-WT-ILK), the kinase-deficient (KD) ILK mutant containing a single mutation at Glu359 for Lys (pFLAG-KD-ILK), the N-terminal deletion (residues 1 to 230; pFLAG-N-ILK), or the mock transfectants pFLAGCMV-2 [15], using Lipofectamine 2000 (Invitrogen Corporation) as specified by the manufacturer. Expression of FLAG-ILK proteins was confirmed by immunofluorescence staining with a mouse monoclonal anti-FLAG antibody (Sigma-Aldrich). After transfection for 24 hours, the cells were fed with fresh selective medium containing G418 geneticin $(800 \mu \mathrm{g} / \mathrm{mL}$; Invitrogen Corporation). Neomycin-resistant clones were cultured in selective medium for another passage and then transferred into Bioflex II six-well plates for experimentation.

\section{Immunofluorescence staining of ACs}

Immunofluorescence staining was performed as described earlier [27]. Briefly, cells were fixed with $2 \%$ paraformaldehyde, permeabilized with $0.2 \%$ Triton $\times 100$ in phosphate-buffered saline, and washed and stained with primary antibodies followed by CY3-labeled secondary antibodies. Beta-actin was stained with fluorescein isothiocyanate-labeled phalloidin (Sigma-Aldrich).

\section{Results}

Mechanical signals induce $A C$ proliferation in the absence or presence of IL-1 $\beta$

To gain insight into the actions of mechanical signals during inflammation, we first determined $\mathrm{AC}$ proliferation in the presence of IL-1 $\beta$. ACs grown on Bioflex plates were mechanoactivated for 90 minutes per day for 2 days with medium alone or medium containing IL-1 $\beta$. On day 3 , spectrophotometric determination of cells by MTT assay revealed that exposure of ACs to mechanical signals significantly upregulated cell proliferation. However, IL-1 $\beta$ significantly suppressed AC proliferation (Figure 1a).

\section{Mechanoactivation of ACs leads to c-Myc, VEGF, and SOX-9 mRNA expression}

VEGF, c-Myc, and SOX-9 are all involved in AC proliferation and differentiation. Therefore, we next determined whether mRNA expression for c-Myc, VEGF, and SOX-9 is upregulated in mechanoactivated ACs in the absence or presence of IL-1 $\beta$. RT-PCR analysis showed that mechanoactivation of ACs significantly upregulated c-Myc, SOX-9, and VEGF mRNA expression involved in AC proliferation and differentiation (Figure 1b-d). We next examined whether ERK1/2 activation was required for the upregulation of mRNA expression for these genes. ACs pretreated for 30 minutes with PD98059 (a MEK1/2and ERK1/2-specific inhibitor) and then exposed to DS showed a significant suppression of DS-induced mRNA expression for c-Myc, SOX-9, and VEGF (Figure 1b-d). 


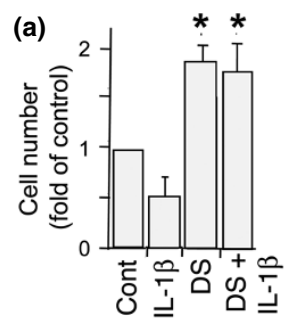

(d)

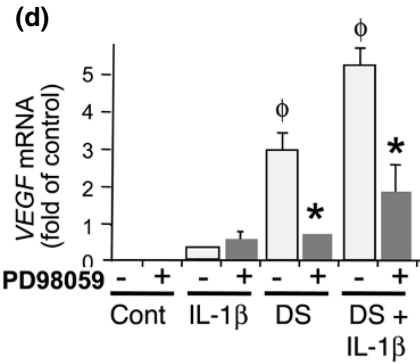

(b)

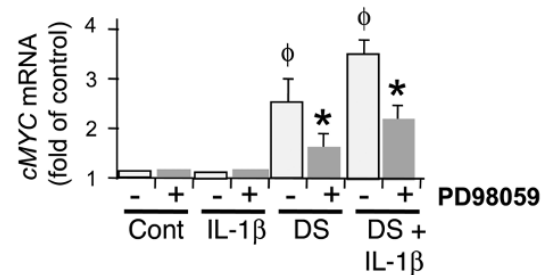

(e)

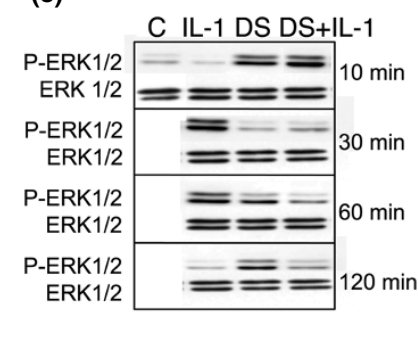

(c)

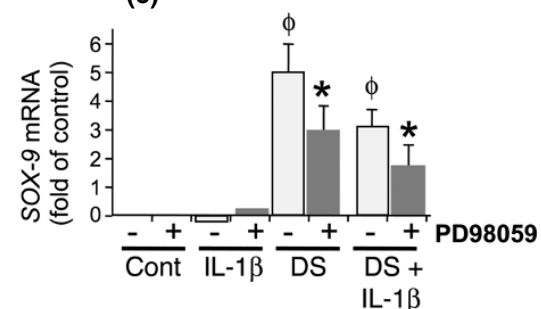

(f)

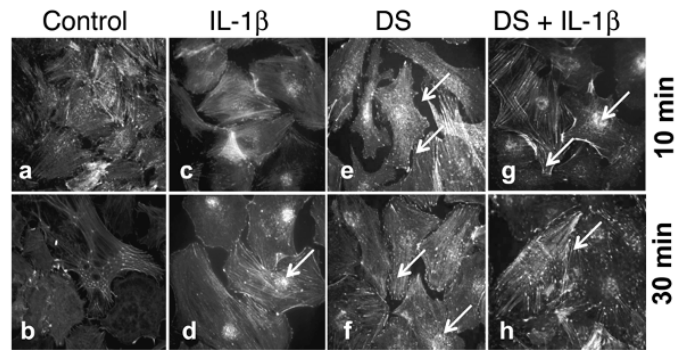

Figure 1 Mechanical signals upregulate articular chondrocyte (AC) proliferation via SOX-9, VEGF, and c-Myc mRNA expression and ERK1/ 2 activation. ACs were exposed to no treatment or to treatment with interleukin-1-beta (IL-1 $\beta$ ), dynamic strain (DS) alone, or DS and IL-1 $\beta$. Subsequently, ACs were subjected to DS for 90 minutes per day for 3 days. (a) On day 4, the rate of cell proliferation was assessed by MTT assay. ACs were treated either with medium alone or with PD98059 $(2 \mu \mathrm{M})$ for 30 minutes. Cells were exposed to the treatment regimens above for 3 hours, and the mRNA expression for c-Myc (b), SOX-9 (c), and VEGF (d) was analyzed by real-time polymerase chain reaction. (e) Western blot analysis showing ERK1/ 2 phosphorylation using phospho-Thr202/Tyr204 ERK1/2 (P-ERK1/2) and total ERK1/2 (T-ERK1/2) antibodies. (f) Immunofluorescence analysis showing minimal phospho-ERK $1 / 2$ in control cells [a], cells stained with secondary antibody alone [b], optimal phosphorylation of ERK1/2 and its nuclear translocation in response to $\mathrm{LL}-1 \beta$ at 10 and 30 minutes $[\mathrm{c}, \mathrm{d}]$, and nuclear translocation and cytoplasmic redistribution of $\mathrm{p}$-ERK1/2 in response to DS in the absence $[\mathrm{e}, \mathrm{f}]$ and presence $[\mathrm{g}, \mathrm{h}]$ of $\mathrm{IL}-1 \beta$. Cells were counterstained with fluorescein isothiocyanate-phalloidin to show $\beta$-actin. Experiments in (a,c-e) were performed in triplicate and were repeated three times in (b) and two times in (f). The error bars represent standard error of the mean (standard error of the mean in a-d). Gels in (e) represent one of three experiments with similar results. ${ }^{\Phi} P<0.05$ as compared with untreated controls; ${ }^{*} P<0.05$ as compared with cells treated with DS or with DS and IL-1. C, control; Cont, control; ERK1/2, extracellular receptor kinase 1/2; MTT, 3-(4,5 dimethylthiazolyl-2)-2,5-diphenyltetrazolium bromide; SOX-9, SRY-related protein-9; VEGF, vascular endothelial cell growth factor.

IL-1 $\beta$ did not induce expression of c-Myc, SOX-9, or VEGF significantly. However, PD98059 significantly abolished DS-dependent c-Myc, SOX-9, and VEGF mRNA induction in the presence of IL- $1 \beta$. These findings suggested that DS induces VEGF and SOX-9 mRNA expression via the ERK1/2 signaling cascade.

\section{Mechanical signals activate ERK1/2 in the absence or presence of IL-1 $\beta$}

Since DS-induced VEGF and SOX-9 were inhibited by PD98059, we next confirmed whether mechanical signals induced ERK1/2 activation. DS significantly upregulated Thr202/Tyr204-ERK1/2 phosphorylation within 10 minutes and was dephosphorylated in the ensuing 20 minutes (Figure 1e). Thereafter, ERK1/2 reactivation was observed at 60 and 120 minutes. In cells treated with IL$1 \beta$, phosphorylation of ERK $1 / 2$ was delayed but sustained between 30 and 60 minutes. More importantly, in cells simultaneously exposed to IL- $1 \beta$ and DS, ERK1/2 was activated within 10 minutes and was subsequently dephosphorylated by 30 minutes. Immunofluorescence staining of ACs revealed that the phosphorylation of ERK1/2 was paralleled by its nuclear translocation and cytoplasmic redistribution in cells treated with DS or with DS and IL-1 $\beta$ (Figure 1f). In cells treated with IL-1 $\beta$, the majority of phospho-ERK1/2 was located in the nuclei at 30 minutes (Figure 1f).

\section{Mechanical signals suppress IL-1 $\beta$-induced B-Raf activation} To understand how mechanical signals sustain their effects in the presence of IL- $1 \beta$, we examined the events upstream of ERK1/2. Western blot analysis using antiphospho-Ser 217/221 MEK1/2 and total MEK1/2 showed that DS induced a rapid and transient phosphorylation of MEK1/2 within 10 minutes. IL-1-induced MEK1/2 activation was observed after 30 minutes of cell activation. Similarly to DS alone, mechanoactivation of cells in the presence of IL-1 $\beta$ showed a rapid and transient phosphorylation of MEK1/2 within 10 minutes (Figure $2 \mathrm{a}$ ).

Since phosphorylation of Raf kinases is necessary for MEK1/2 activation, we next determined whether A-Raf, B-Raf, or c-Raf is activated by DS. DS or IL- $1 \beta$ did not activate A-Raf (data not shown). DS alone or in the presence of IL-1 $\beta$ induced a rapid phosphorylation of Ser338 on c-Raf (Figure 2b). B-Raf was constitutively phosphorylated in ACs. Western blot analysis demonstrated that IL- 


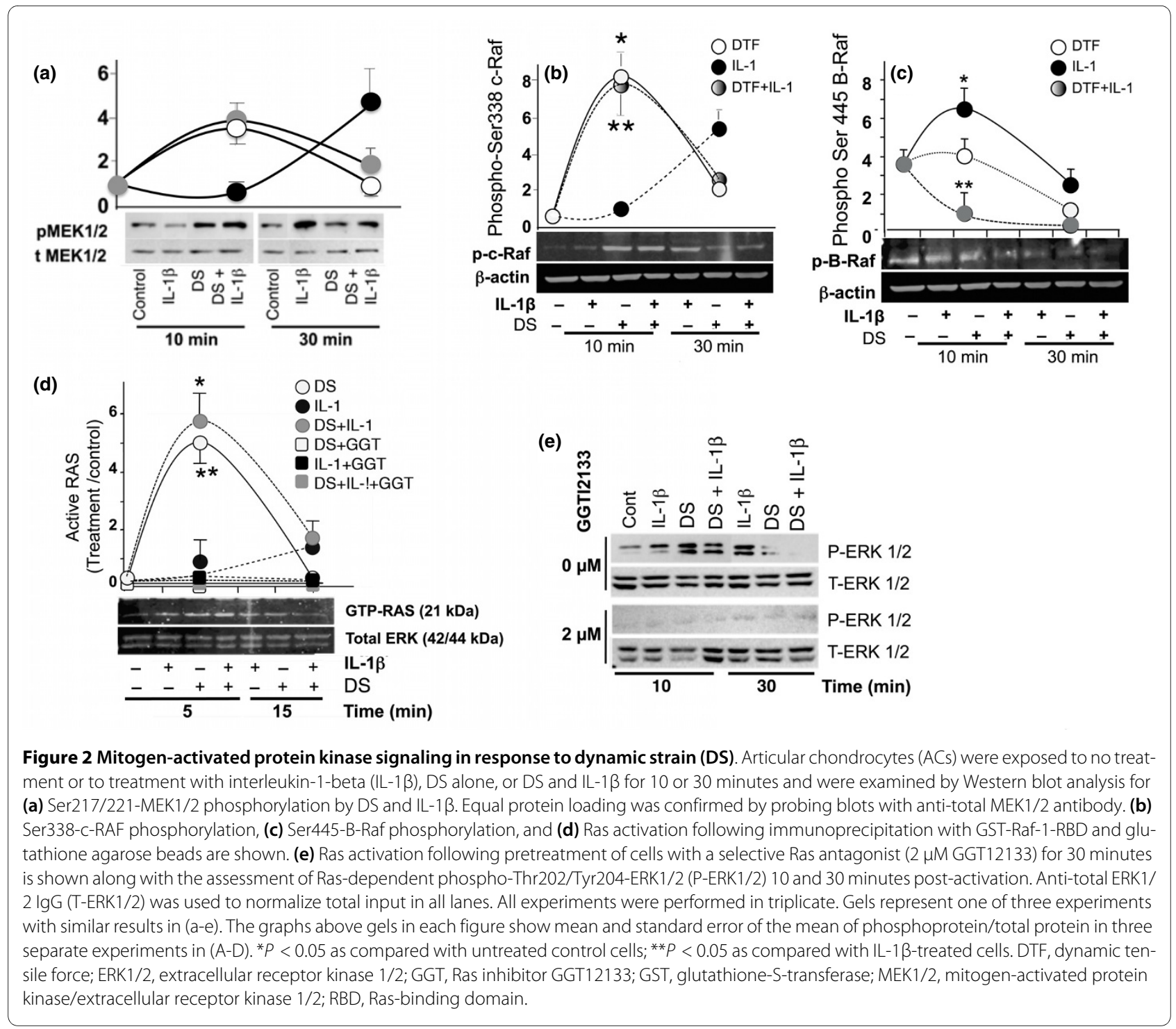

$1 \beta$ significantly activated B-Raf by phosphorylating its Ser445 residues. However, B-Raf was not activated by DS but it did suppress IL-1 $\beta$-induced Ser445-B-Raf phosphorylation (Figure 2c).

Using a similar experimental strategy, we next examined the activation of the RAS proteins. RAS proteins are found as GTP-bound active and GDP-bound inactive forms. ACs exposed to the above experimental regimens were lysed and subjected to precipitation to capture activated RAS with GST-Raf-RBD and glutathione agarose beads. Western blot analysis revealed that DS alone or in the presence of IL-1 $\beta$ induced a rapid but transient activation of RAS within 5 minutes (Figure $2 \mathrm{~d}$ ). However, IL$1 \beta$ induced a minimal RAS activation. Untreated ACs exhibited negligible GTP-bound activated RAS. To confirm these observations, ACs were further pretreated with a selective antagonist of RAS, GGT12133 (2 or 10 $\mu \mathrm{M})$, and subsequently stimulated for 5 or 15 minutes.
GGT12133 (2 $\mu \mathrm{M})$ completely inhibited DS-induced ERK1/2 activation, confirming that mechanical signals induce RAS activation in the absence or presence of an inflammatory stimulus (Figure 2e).

\section{Mechanical signals activate ILK to initiate ERK1/2 signaling cascade}

ILK is shown to activate RAS proteins. To determine whether ILK activation was necessary for mechanoactivation-induced RAS activation, ACs were transfected with plasmids containing FLAG-ILK expression vectors containing the full-length ILK (FLAG-WT-ILK), truncated $\mathrm{N}$ terminal (residues 1-230, FLAG-N-ILK), and the KD ILK mutant (FLAG-KD-ILK) containing a single mutation (Glu359 at Lys) or with pFLAG-CMV-2 vector lacking the ILK sequence as a control $[28,29]$. ACs shown in Figure 3a were untransfected $(\mathrm{a}, \mathrm{b})$ or were transfected with FLAG-CMV-2 empty vector (c,d), FLAG-KD-ILK 
(a)
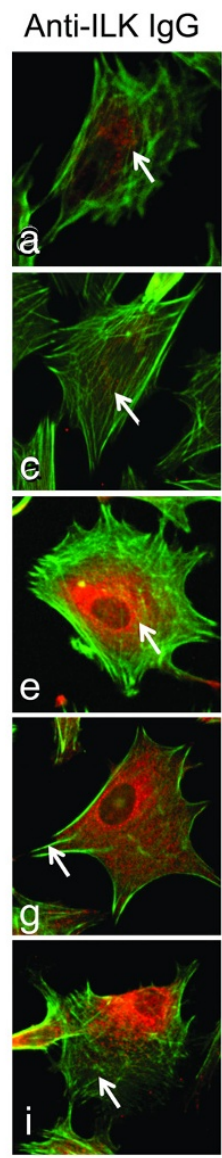

Anti-FLAG IgG
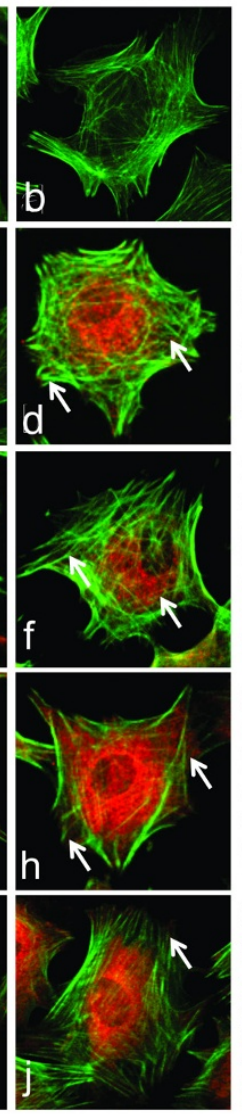

(b)

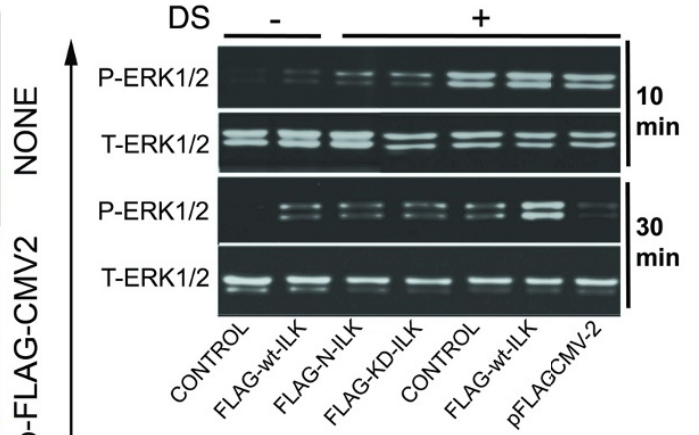

(d)

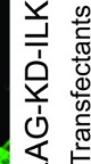

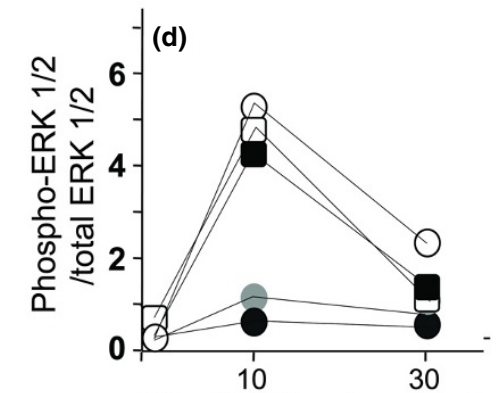

$\mathrm{DS}+\mathrm{IL}-1 \beta$ treatment $(\mathrm{min})$

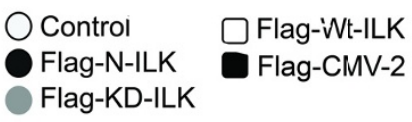

(c)

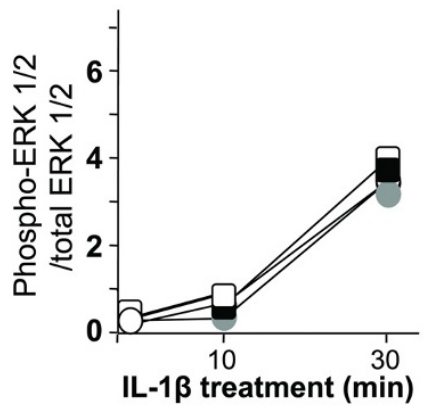

Figure 3 Mechanical signals activate integrin-linked kinase (ILK). (a) Articular chondrocytes (ACs) either were not transfected [a,b] or were transfected with p-FLAG-CMV2 empty [c,d], FLAG-KD-ILK [e,f], FLAG-N-ILK [g,h], or FLAG-WT ILK [i,j]. ACs were immunostained with anti-ILK (left frames) or anti-FLAG (right frames) antibodies and CY3-conjugated secondary antibodies. All cells were counterstained with fluorescein isothiocyanate-phalloidin to visualize $\beta$-actin. Western blot analysis shows ERK1/2 activation in untransfected ACs or those transfected with FLAG-N-ILK, FLAG-KD-ILK, FLAGWT-ILK, or pFLAG-CMV2 exposed to (b) no strain or dynamic strain (DS) alone, (c) interleukin-1-beta (IL-1 1 ) alone, or (d) DS and IL-1 $1 \beta$. Frames (c,d) show semiquantitative estimation of bands in Western blots. All figures represent one of three similar experiments. ERK1/2, extracellular receptor kinase 1/2; FLAG, polypeptide protein sequence DYKDDDDK; KD, kinase-deficient; P-ERK1/2, phospho-Thr202/Tyr204-extracellular receptor kinase 1/2; T-ERK1/2, total extracellular receptor kinase 1/2; WT, wild-type.

$(\mathrm{e}, \mathrm{f})$, mutant FLAG-N-ILK (g,h), or FLAG-WT-ILK $(\mathrm{i}, \mathrm{j})$, and ILK was detected by rabbit anti-ILK (a,c,e,g,i) or rabbit anti-FLAG antibodies (b,d,f,h,j). Cells stained with goat anti-rabbit CY3-labeled secondary antibodies alone did not show staining (data not shown).

Western blot analysis showed that untransfected control cells and those transfected with FLAG-WT-ILK did not exhibit constitutive ERK1/2 phosphorylation (Figure $3 \mathrm{~b})$. However, within 10 minutes, exposure of untransfected control cells and cells transfected with pFLAGCMV-2 or FLAG-WT-ILK to DS showed ERK1/2 phosphorylation, which remained high in cells overexpressing WT-ILK. However, mechanoactivation of ACs transfected with FLAG-N-ILK or FLAG-KD-ILK failed to induce ERK1/2 phosphorylation in cells (Figure 3b). Densitometric analysis of the same samples probed with anti- total ERK1/2 antibody confirmed equal protein input in all lanes (Figure 3b). ACs activated by IL-1 $\beta$ showed ERK1/2 activation in cells transfected with FLAGmutant-ILK or FLAG-WT-ILK following 30 minutes of activation (Figure 3c). However, cells simultaneously activated with IL- $1 \beta$ and DS showed ERK1/2 activation in only the untransfected cells or those transfected with plasmids containing FLAG-WT-ILK or pFLAG-CMV-2 (Figure 3d).

\section{Discussion}

We have shown that dynamic mechanical signals vitally control AC proliferation and differentiation by regulating the MAPK signaling cascade. Furthermore, the actions of mechanical signals are sustained in the presence of proinflammatory signals induced by IL- $1 \beta$. We have exposed 
ACs to dynamic tensile forces to assess their potential in controlling cell growth. During joint movement, ACs simultaneously experience dynamic compression-, tension-, and torsion-induced forces. In vitro, ACs subjected to $10 \%$ compression in three-dimensional microfiber or agarose constructs exhibit many biochemical changes similar to those of ACs exposed to $6 \%$ tensile forces. For example, $10 \%$ compressive forces as well as $6 \%$ tensile forces suppress proinflammatory gene induction, upregulate total proteoglycan contents, and aggrecan, collagen type II, and SOX-9 mRNA induction in ACs [7,8,30-32]. Therefore, in this study, $6 \%$ tensile forces were used to examine the signaling events induced by DS. However, so far, the extent of compressive or tensile forces experienced by ACs during joint movement in vivo is not clear.

Intracellular signal transduction by mechanical signals begins with ILK activation. This was evident by the observations that mechanical signals failed to induce ERK1/2 phosphorylation in ACs transfected with mutant-ILK or kinase activity-deficient ILK plasmids. However, mechanical signals induced ERK1/2 activation in ACs transfected with WT ILK or untransfected cells. These studies revealed that ILK activation by mechanical signals is of critical importance given the fact that integrins are the putative mechanosensors of chondrocytes, and ILK is one of the central signaling components of the integrin complex [15]. Interestingly, mechanical signals are also perceived via integrins to activate Rho GTPases to regulate cytoskeletal rearrangements [33]. This indicates that mechanical signals regulate diverse cellular functions via integrin engagement.

Mechanoactivation of ACs leads to the rapid activation of RAS. In an effort to examine whether mechanical signals regulate RAS during inflammation, we examined the effects of IL- $1 \beta$ on RAS activation. IL- $1 \beta$ induces minimal activation of RAS. Nevertheless, RAS activation is similar in mechanoactivated cells irrespectively of the presence of IL-1 $\beta$. RAS activation is associated with ERK1/2-mediated cell proliferation [34]. Consistent with these findings, our data show that the RAS inhibitor GGT12133 attenuates ERK1/2 phosphorylation induced by mechanical signals. RAS activation is central to activation of many cell surface receptors, such as growth factor receptors, receptor tyrosine kinases, integrins, and IL-6 receptors [34-36], further suggesting that dynamic mechanical signals activate signaling molecules similar to other growth factors.

To examine how mechanical signals and IL-1 $\beta$ regulate ERK1/2 signaling cascade that result in differential gene expression, we next examined the activation of Rafs [37]. Mechanical signals trigger c-Raf kinase activity by phosphorylating Ser338 residues. However, IL-1 $\beta$ induces Ser445-B-Raf phosphorylation. B-Raf was not activated by mechanical signals. However, mechanical signals inhibited IL-1 $\beta$-induced B-Raf activation. This disparity in the activation of Rafs may play a critical role in the differential processing of signals generated by IL- $1 \beta$ and mechanical forces. However, the mechanisms that underlie this regulation of c-Raf and B-Raf remain to be elucidated.

Activation of B-Raf by IL- $1 \beta$ or c-Raf by mechanical signals results in MEK1/2 activation via Ser217/221 phosphorylation [19]. Subsequently, MEK1/2 activates ERK1/ 2 by phosphorylating both Thr202/Tyr204 residues. Following mechanoactivation, phosphorylated ERK1/2 rapidly translocates to the nucleus and is redistributed to the cell surface. ERK proteins after activation translocate to the nuclear compartment, where they act as the main executor of ERK1/2 biological functions, and channel a diverse array of signals via downstream targets. Additionally, ERK dimers and scaffolds translocate to cognate cytoplasmic substrates, where they stabilize ERK1/2 and Myc functions in cell proliferation [35,38,39].

Interestingly, ERK $1 / 2$ activation is temporally regulated in response to DS as well as IL- $1 \beta$. DS rapidly induces ERK1/2 phosphorylation, which is observed within 10 minutes. IL-1 $\beta$-induced ERK1/2 phosphorylation is apparent at 30 minutes. It is likely that DS, by activating kinases upstream of ERK1/2, initiates a feedback loop that suppresses IL-1 $\beta$-induced ERK1/2 activation. Such early activation of ERK1/2 by DS may likely play a role in sustaining its effects in the presence of IL-1 $\beta$.

Mechanoactivation of ACs leads to c-Myc, VEGF, and SOX-9 mRNA expressions, all of which have been implicated in the proliferative response of cells to a variety of stimuli [35,40]. Furthermore, ERK1/2 activation is required for c-Myc, SOX-9, and VEGF mRNA expression, as evidenced by the suppression of their transcriptional activation by PD98059. We have also observed that ERK1/2 activation by IL-1 $\beta$ fails to induce SOX-9 or VEGF expression. This may explain the suppression of AC proliferation in the presence IL-1 $\beta$. These findings again point to similarities between mechanical signals and other growth factors that use the ERK $1 / 2 / \mathrm{Myc}$ signaling cascade to regulate cell proliferation [23,36,41]. Furthermore, the fact that mechanical signals upregulate $c-M y c$, SOX-9, and VEGF in the presence of IL- $1 \beta$ supports the benefits of mechanoactivation of ACs in the inflamed cartilage.

\section{Conclusions}

Our findings demonstrate for the first time that mechanical signals suppress the ERK1/2 signaling cascade of IL$1 \beta$, indicating a critical role for these signals in rescuing cartilage from the detrimental effects of IL- $1 \beta$ during inflammation. The cellular decision-making in response to mechanical forces occurs swiftly and is phosphorelayed via ILK to downstream signaling targets. None- 
theless, activation of intermediate signaling molecules like c-Raf and B-Raf may be critical in regulating ERK1/2 transcriptional activity in response to mechanosignaling. Only c-Raf is activated by mechanical signals but it inhibits B-Raf activation by IL- $1 \beta$. Activated hetrodimers and homodimers of B-Raf and c-Raf regulate downstream activation of MAPKs. By suppressing B-Raf activation, mechanical signals may likely alter a critical event important for the downstream IL-1 $\beta$ signaling. This may lead to the SOX-9, VEGF, and Myc upregulation responsible for cell proliferation in IL-1 $\beta$-treated cells. Earlier studies have shown that mechanical signals also suppress inflammation by inhibiting nuclear factor-kappa-B activation and thus expression of proinflammatory genes, such as IL-1 $\beta$, TNF- $\alpha$, inducible nitric oxide synthase, matrix metalloproteinases, and lipopolysaccharide $[3,7,8,25]$. The present findings thus demonstrate, at least in part, the basis for the regenerative potential of mechanical signals in arthritic diseases. Furthermore, studies show the importance of the ERK $1 / 2$ signaling cascade in mediating proliferative actions of mechanical signals in proinflammatory environments.

\begin{abstract}
Abbreviations
AC: articular chondrocyte; DS: dynamic strain; ERK1/2: extracellular receptor kinase 1/2; FBS: fetal bovine serum; FLAG: polypeptide protein sequence DYKDDDDK; GDP: guanosine diphosphate; GST: glutathione-S-transferase; GTP: guanosine triphosphate; HRP: horseradish peroxidase; IL-1: interleukin-1; ILK: integrin-linked kinase; KD: kinase-deficient; MAPK: mitogen-activated protein kinase; MEK1/2: mitogen-activated protein kinase/extracellular receptor kinase 1/2; MTT: 3-(4,5 dimethylthiazolyl-2)-2,5-diphenyltetrazolium bromide; RAF: proto-oncogene c-RAF kinase; RBD: Ras-binding domain; RT-PCR: real-time polymerase chain reaction; SOX-9: SRY-related protein-9; TCM: tissue culture medium; TNF-a: tumor necrosis factor-alpha; VEGF: vascular endothelial cell growth factor; WT: wild-type.
\end{abstract}

\section{Competing interests}

The authors declare that they have no competing interests.

\section{Authors' contributions}

PMP carried out Ras activation, AC isolation, DS exposure, and SOX-9, VEGF, and Myc induction. EW performed ERK and MAPK activation. SM carried out Raf/Ras activation. BR-D carried out activation and expression of ERK-responsive transcription factors. JL was responsible for immunofluorescence. JN performed stretch protocol optimization. BR carried out ILK activation. YH was responsible for activation of transcription factors. JD conducted experimental planning and data management. NP prepared the manuscript. CW prepared ILK constructs. SA conducted experimental planning and prepared the manuscript. All authors read and approved the final manuscript.

\section{Acknowledgements}

This work was supported by AR048781 and DE15399.

\footnotetext{
Author Details

'Biomechanics and Tissue Engineering Laboratory, The Ohio State University, Postle Hall, 305 W 12th Avenue, Columbus, OH 43210, USA, 2Department of Orthodontics, University of Bonn, Welschnonnenstrasse 17, 53111 Bonn, Germany, ${ }^{3}$ Department of Orthopedics, University of Regensburg, Kaiser-Karl V-Allee 3, 93077 Bad Abbach, Germany, ${ }^{4}$ Department of Periodontics, University of Bonn, Welschnonnenstrasse 17, 53111 Bonn, Germany, ${ }^{5}$ Department of Oral Biology, School of Dental Medicine, Salk Hall, 3501 Terrace Street, University of Pittsburgh, Pittsburgh, PA 15261, USA and ${ }^{6}$ Department of Pathology, University of Pittsburgh, School of Medicine, S-417 BST, 200 Lothrop Street, Pittsburgh, PA 15261, USA
}

Received: 29 December 2009 Revised: 24 March 2010

Accepted: 28 May 2010 Published: 28 May 2010

\section{References}

1. Zuscik MJ, Hilton MJ, Zhang X, Chen D, O'Keefe RJ: Regulation of chondrogenesis and chondrocyte differentiation by stress. J Clin Invest 2008, 118:429-438.

2. Chiquet $M$, Gelman $L$, Lutz R, Maier S: From mechanotransduction to extracellular matrix gene expression in fibroblasts. Biochim Biophys Acta 2009, 1793:911-920.

3. Dossumbekova A, Anghelina M, Madhavan S, He L, Quan N, Knobloch T, Agarwal S: Biomechanical signals inhibit IKK activity to attenuate NFkappaB transcription activity in inflamed chondrocytes. Arthritis Rheum 2007, 56:3284-3296.

4. Agarwal S, Deschner J, Long P, Verma A, Hofman C, Evans CH, Piesco N: Role of NF-kappaB transcription factors in antiinflammatory and proinflammatory actions of mechanical signals. Arthritis Rheum 2004, 50:3541-3548

5. Wang B, Yang $Y$, Friedman PA: $\mathrm{Na} / \mathrm{H}$ exchange regulatory factor 1 , a novel AKT-associating protein, regulates extracellular signal-regulated kinase signaling through a B-raf-mediated pathway. Mol Biol Cell 2008, 19:1637-1645.

6. Nugent GE, Aneloski NM, Schmidt TA, Schumacher BL, Voegtline MS, Sah RL: Dynamic shear stimulation of bovine cartilage biosynthesis of proteoglycan 4. Arthritis Rheum 2006, 54:1888-1896.

7. McNulty AL, Estes BT, Wilusz RE, Weinberg JB, Guilak F: Dynamic loading enhances integrative meniscal repair in the presence of interleukin-1. Osteoarthritis Cartilage 2010, 18:830-838.

8. Nam J, Aguda B, Agarwal S: Biomechanical thresholds regulate inflammation through the NF-KB pathway: experiments and modeling. PLOS ONE 2009, 4:e5262.

9. Chowdhury TT, Bader DL, Lee DA: Dynamic compression counteracts IL1 beta-induced release of nitric oxide and PGE2 by superficial zone chondrocytes cultured in agarose constructs. Osteoarthritis Cartilage 2003, 11:688-696.

10. Wang N, Tytell JD, Ingber DE: Mechanotransduction at a distance: mechanically coupling the extracellular matrix with the nucleus. Nat Rev Mol Cell Biol 2009, 10:75-82.

11. Na S, Collin O, Chowdhury F, Tay B, Ouyang M, Wang Y, Wang N: Rapid signal transduction in living cells is a unique feature of mechanotransduction. Proc Natl Acad Sci USA 2008, 105:6626-6631.

12. Ryan JA, Eisner EA, Duraine G, You Z, Hari Reddi A: Mechanical compression of articular cartilage induces chondrocyte proliferation and inhibits proteoglycan synthesis by activation of the ERK pathway: implications for tissue engineering and regenerative medicine. J Tissue Eng Regen Med 2009, 3:107-116.

13. Raducanu A, Hunziker EB, Drosse I, Aszodi A: \{Beta\}1 integrin deficiency results in multiple abnormalities of the knee joint. J Biol Chem 2009, 284:23780-23792.

14. Maier S, Lutz R, Gelman L, Sarasa-Renedo A, Schenk S, Grashoff C, Chiquet $M$ : Tenascin- $C$ induction by cyclic strain requires integrin-linked kinase. Biochim Biophys Acta 2008, 1783:1150-1162.

15. WU C: PINCH, N(i)ck and the ILK: network wiring at cell-matrix adhesions. Trends Cell Biol 2005, 15:460-466.

16. Mammoto A, Connor KM, Mammoto T, Yung CW, Huh D, Aderman CM, Mostoslavsky G, Smith LE, Ingber DE: A mechanosensitive transcriptional mechanism that controls angiogenesis. Nature 2009, 457:1 103-1108

17. Gray DS, Liu WF, Shen CJ, Bhadriraju K, Nelson CM, Chen CS: Engineering amount of cell-cell contact demonstrates biphasic proliferative regulation through RhoA and the actin cytoskeleton. Exp Cell Res 2008, 314:2846-2854.

18. McKay MM, Morrison DK: Integrating signals from RTKs to ERK/MAPK. Oncogene 2007, 26:3113-3121.

19. Torii S, Nakayama K, Yamamoto T, Nishida E: Regulatory mechanisms and function of ERK MAP kinases. J Biochem 2004, 136:557-561.

20. Zhang M, Zhou Q, Liang QQ, Li CG, Holz JD, Tang D, Sheu TJ, Li TF, Shi Q, Wang YJ: IGF-1 regulation of type II collagen and MMP-13 expression in rat endplate chondrocytes via distinct signaling pathways. Osteoarthritis Cartilage 2009, 17:100-106.

21. Jiang $B$, Brecher $P$, Cohen RA: Persistent activation of nuclear factorkappaB by interleukin-1 beta and subsequent inducible NO synthase 
expression requires extracellular signal-regulated kinase. Arterioscler Thromb Vasc Biol 2001, 21:1915-1920.

22. Ory S, Morrison DK: Signal transduction: implications for ras-dependent ERK signaling. Curr Biol 2004, 14:R277-278.

23. Kolettas E, Muir HI, Barrett JC, Hardingham TE: Chondrocyte phenotype and cell survival are regulated by culture conditions and by specific cytokines through the expression of sox-9 transcription factor. Rheumatology (Oxford) 2001, 40:1146-1156.

24. Li KW, Wang AS, Sah RL: Microenvironment regulation of extracellular signal-regulated kinase activity in chondrocytes: effects of culture configuration, interleukin-1, and compressive stress. Arthritis Rheum 2003, 48:689-699.

25. Madhavan S, Anghelina M, Sjostrom D, Dossumbekova A, Guttridge DC Agarwal S: Biomechanical signals suppress TAK1 activation to inhibit $\mathrm{NF}$-\{kappa\}B transcriptional activation in fibrochondrocytes. J Immunol 2007, 179:6246-6254.

26. Livak KJ, Schmittgen TD: Analysis of relative gene expression data using real-time quantitative PCR and the 2(-delta delta $C(T)$ ) method. Methods 2001, 25:402-408

27. Li F, Zhang Y, Wu C: Integrin-linked kinase is localized to cell-matrix focal adhesions but not cell-cell adhesion sites and the focal adhesion localization of integrin-linked kinase is regulated by the PINCHbinding ANK repeats. J Cell Sci 1999, 112(Pt 24):4589-4599.

28. Li Y, Yang J, Dai C, Wu C, Liu Y: Role for integrin-linked kinase in mediating tubular epithelial to mesenchymal transition and renal interstitial fibrogenesis. J Clin Invest 2003, 112:503-516.

29. Wu C, Dedhar S: Integrin-linked kinase (ILK) and its interactors: a new paradigm for the coupling of extracellular matrix to actin cytoskeleton and signaling complexes. J Cell Biol 2001, 155:505-510.

30. Chowdhury TT, Arghandawi S, Brand J, Akanji OO, Bader DL, Salter DM, Lee DA: Dynamic compression counteracts IL-1 beta induced inducible nitric oxide synthase and cyclo-oxygenase-2 expression in chondrocyte/agarose constructs. Arthritis Res Ther 2008, 10:R35.

31. Nam J, Rath B, Knobloch T, Lannutti JJ, Agarwal S: Novel electrospun scaffolds for the molecular analysis of chondrocytes under dynamic compression. Tissue Eng Part A 2009, 15:513-523.

32. Deschner J, Hofman CR, Piesco NP, Agarwal S: Signal transduction by mechanical strain in chondrocytes. Curr Opin Clin Nutr Metab Care 2003, 6:289-293.

33. Liu WF, Nelson CM, Tan JL, Chen CS: Cadherins, RhoA, and Rac1 are differentially required for stretch-mediated proliferation in endothelial versus smooth muscle cells. Circ Res 2007, 101:e44-52

34. Kiel C, Serrano L: Cell type-specific importance of ras-c-raf complex association rate constants for MAPK signaling. Sci Signal 2009, 2:ra38.

35. Chambard JC, Lefloch R, Pouyssegur J, Lenormand P: ERK implication in cell cycle regulation. Biochim Biophys Acta 2007, 1773:1299-1310.

36. Bluteau G, Julien M, Magne D, Mallein-Gerin F, Weiss P, Daculsi G, Guicheux J: VEGF and VEGF receptors are differentially expressed in chondrocytes. Bone 2007, 40:568-576.

37. Molina JR, Adjei AA: The Ras/Raf/MAPK pathway. J Thorac Oncol 2006, 1:7-9.

38. Lee T, Yao G, Nevins J, You L: Sensing and integration of erk and PI3K signals by myc. PLoS Comput Biol 2008, 4:e1000013.

39. Sears R, Nuckolls F, Haura E, Taya Y, Tamai K, Nevins JR: Multiple rasdependent phosphorylation pathways regulate myc protein stability. Genes Dev 2000, 14:2501-2514

40. Malumbres M, Pellicer A: RAS pathways to cell cycle control and cell transformation. Front Biosci 1998, 3:d887-912.

41. Tew SR, Hardingham TE: Regulation of SOX9 mRNA in human articular chondrocytes involving p38 MAPK activation and mRNA stabilization J Biol Chem 2006, 281:39471-39479.

doi: $10.1186 / a r 3039$

Cite this article as: Perera et al., Mechanical signals control SOX-9, VEGF, and c-Myc expression and cell proliferation during inflammation via integrinlinked kinase, B-Raf, and ERK1/2-dependent signaling in articular chondrocytes Arthritis Research \& Therapy 2010, 12:R106

\section{Submit your next manuscript to BioMed Central} and take full advantage of:

- Convenient online submission

- Thorough peer review

- No space constraints or color figure charges

- Immediate publication on acceptance

- Inclusion in PubMed, CAS, Scopus and Google Scholar

- Research which is freely available for redistribution 